\title{
A signature of seven immune-related genes predicts overall survival in male gastric cancer patients
}

\author{
Xin $X u^{1,2}$, Yida $\mathrm{Lu}^{1,2}$, Youliang Wu ${ }^{1,2}$, Mingliang Wang ${ }^{1,2}$, Xiaodong Wang ${ }^{1,2}$, Huizhen Wang ${ }^{1}$, Bo Chen ${ }^{1}$ \\ and Yongxiang $\mathrm{Li}^{1 *}$
}

\begin{abstract}
Background: Gastric cancer (GC) has a high mortality rate and is one of the most fatal malignant tumours. Male sex has been proven as an independent risk factor for GC. This study aimed to identify immune-related genes (IRGs) associated with the prognosis of male GC.
\end{abstract}

Methods: RNA sequencing and clinical data were obtained from The Cancer Genome Atlas (TCGA) database. Differentially expressed IRGs between male GC and normal tissues were identified by integrated bioinformatics analysis. Univariate and multivariate Cox regression analyses were applied to screen survival-associated IRGs. Then, GC patients were separated into high- and low-risk groups based on the median risk score. Furthermore, a nomogram was constructed based on the TCGA dataset. The prognostic value of the risk signature model was evaluated by Kaplan-Meier curve, receiver operating characteristic (ROC), Harrell's concordance index and calibration curves. In addition, the gene expression dataset from the Gene Expression Omnibus (GEO) was also downloaded for external validation. The relative proportions of 22 types of infiltrating immune cells in each male GC sample were evaluated using CIBERSORT.

Results: A total of 276 differentially expressed IRGs were screened, including 189 up-regulated and 87 down-regulated genes. Subsequently, a seven-IRGs signature (LCN12, CCL21, RNASE2, CGB5, NRG4, AGTR1 and NPR3) was identified to be significantly associated with the overall survival (OS) of male GC patients. Survival analysis indicated that patients in the high-risk group exhibited a poor clinical outcome. The results of multivariate analysis revealed that the risk score was an independent prognostic factor. The established nomogram could be used to evaluate the prognosis of individual male GC patients. Further analysis showed that the prognostic model had excellent predictive performance in both TCGA and validated cohorts. Besides, the results of tumour-infiltrating immune cell analysis indicated that the seven-IRGs signature could reflect the status of the tumour immune microenvironment.

Conclusions: Our study developed a novel seven-IRGs risk signature for individualized survival prediction of male GC patients.

Keywords: Immune-related genes, Prognosis, The Cancer Genome Atlas, Gastric cancer, Male, Overall survival

*Correspondence: liyongxiang@ahmu.edu.cn

${ }^{1}$ Department of General Surgery, The First Affiliated Hospital of Anhui Medical University, 218 JiXi Avenue, Hefei 230022, Anhui, China

Full list of author information is available at the end of the article

\section{Background}

Epidemiological evidence indicates that the incidence of gastric cancer (GC) in males is nearly two times of that in females [1]. Moreover, male patients with GC always have a higher tumour-node-metastasis (TNM) stage and a poorer prognosis than their female counterparts [2]. Recently, it has been strongly recommended that 
sex be examined as a biological variable in future cancer research due to its substantial influence on the occurrence and development of tumours [3]. Existing widely utilized models or biomarkers may not properly predict prognosis for male GC patients. Hence, it is urgently necessary to identify novel biomarkers for survival prediction in male GC patients.

As one of the fundamental hallmarks of cancer [4], evading immune surveillance has been increasingly appreciated in recent years. The immunotherapy targeting immune checkpoint has achieved impressive success in the treatment of several tumours [5, 6]. Growing evidence indicates that tumour-infiltrating immune cells play an important role in cancer initiation and progression and have been proposed to be valuable for the diagnosis and prognosis of tumours [7-9]. In addition, immune-related genes (IRGs) within the tumour microenvironment (TME) also represent tremendous potential value in serving as prognostic biomarkers $[10,11]$. Recent studies have integrated IRG expression profiles with clinical information to gain insight into the potential clinical utility of IRGs in risk stratification and survival prediction in several tumours [12-15]. In terms of the prognostic value of IRGs in GC, Jiang et al. proposed a 16-IRGs signature that could serve as a reliable prognostic tool for overall survival (OS) [16]. Nevertheless, IRGs prognostic models for male GC patients have not been reported.

This study is the first to identify novel IRGs associated with OS. We proposed an individualized prognostic model for male GC patients by using IRGs expression profiles and clinical data from The Cancer Genome Atlas (TCGA). Then, we integrated the prognostic model and clinical pathological parameters to establish a nomogram for predicting outcome. The prognostic value of IRGs signature was further validated in GSE15460 from the Gene Expression Omnibus (GEO) database. In addition, CIBERSORT was applied to elucidate the correlation between the risk signature and the abundances of infiltrative immune cells in male GC patients. We aim to provide a new insight into predicting prognosis for male $\mathrm{GC}$ patients.

\section{Materials and methods}

\section{Online databases}

The RNA-Seq data and clinical information of male GC patients were obtained from TCGA database (https:// tcga-data.nci.nih.gov/tcga/). A total of 2498 IRGs were obtained from the Immunology Database and Analysis Portal (ImmPort) database (https://www.immport.org/ home) [17]. We also downloaded gene expression profiles and clinical data of GSE15460 from GEO (http://www. ncbi.nlm.nih.gov/geo/) for validation dataset.

\section{Differentially expressed IRGs and enrichment analysis}

The differential expression of IRGs between male GC and their non-tumour counterparts was identified using package "limma" in R software [18], with the cutoff value of $\mid \log 2$ fold change (FC) $\mid>1$ and false discovery rate $(\mathrm{FDR})<0.05$. Gene Ontology $(\mathrm{GO})$ and Kyoto Encyclopedia of Genes and Genomes (KEGG) enrichment analyses were conducted using the $\mathrm{R}$ software "clusterprofiler" package to explore potential molecular mechanisms of the differentially expressed IRGs. The "ggplot2" and "GOplot" packages in R were used for visualization of GO and KEGG enrichment analysis results.

\section{Establishment of the prognostic IRGs signature}

To improve the predictive accuracy of IRGs signature, only male GC patients with a follow-up time of more than 60 days were included in our study. Univariate and multivariate Cox regression analysis were performed to search for OS-related IRGs using "survival" package in $\mathrm{R}$. The risk score of each male GC patient was calculated with the following formula: risk score $=$ expression level of $\mathrm{IRG}_{1} \times \beta_{1}+$ expression level of $\mathrm{IRG}_{2} \times \beta_{2}$ $+\ldots+$ expression level of $\mathrm{IRG}_{\mathrm{n}} \times \beta_{\mathrm{n}}$; where $\beta$ is the coefficient calculated by the multivariate Cox regression model [19]. Subsequently, male GC patients were divided into high- and low-risk groups according to the median risk score. The time-dependent receiver operating characteristic (ROC) curve was constructed to evaluate the predictive value of the prognostic IRGs signature for OS using the R software package "survivalROC". By using the R package "survival", the KaplanMeier survival curve was drawn to estimate the OS difference in the high- and low-risk groups, with statistical significance evaluated by the log-rank test. Moreover, to determine whether the prognostic IRGs signature could be an independent predictor of OS in male GC patients, univariate and multivariate Cox regression analyses were performed. Age, grade, stage, $\mathrm{T}$ stage, $\mathrm{N}$ stage, $\mathrm{M}$ stage, and risk score were employed as covariates.

\section{Construction of a prognostic nomogram}

We formulated a nomogram on the basis of risk score and clinical parameters to assess the probability of 1-, 3-, and 5-year OS for male GC patients using the "rms," "foreign," and "survival" packages in R. The concordance index (C-index) was calculated to assess the performance of the prognostic nomogram. Calibration plots were also drawn to estimate the consistency between actual and predicted survival. 


\section{External validation of IRGs signature}

The risk score for each enrolled patient in the validation dataset was calculated with the same constructed formula based on the prognostic IRGs signature model. Similarly, male GC patients were divided into high- and low-risk groups according to the median risk score. Then, Kaplan-Meier curves for the high- and low-risk groups combined with log-rank test were used to assess the predictive value of the prognostic IRGs signature. Survival ROC curves were applied to assess the predictive power of the model. In addition, the calibration plots for survival probability at 3- or 5-year were generated to evaluate the prognostic accuracy of the nomogram.

\section{Tumour-infiltrating immune cell analysis}

CIBERSORT, a newly developed deconvolution algorithm [20], was utilized to determine the relative proportions of 22 types of infiltrating immune cells in each male GC sample. Subsequently, a Wilcoxon rank-sum test was applied to evaluate the difference in the abundance of immune cells between the high-and low-risk groups, and illustrated by the "fmsb" packages in R.

\section{Statistical analysis}

The statistical analyses were performed using R software (version 3.6.3) and GraphPad Prism 6 (GraphPad Software Inc., USA). Kaplan-Meier curves and the log-rank test were applied to assess the statistical significance of the survival rates between the high- and low-risk groups. Univariate and multivariate Cox regression analyses were performed to evaluate significant prognostic factors. The Wilcoxon rank-sum test was used to test the statistical significance between high- and low-risk groups. Data were presented as mean $\pm \mathrm{SD}$ and $P<0.05$ was considered significant.

\section{Results \\ Identification and enrichment analysis of differentially expressed IRGs}

The RNA-Seq data from TCGA consisted of 263 cases, including 241 male GC cases and 22 male non-tumour cases. Compared to male non-tumour tissues, a total of 276 differentially expressed IRGs including 87 downregulated and 189 upregulated were screened out, with the cut-off criteria of $\mid \log 2$ fold change (FC) $\mid>1$ and false discovery rate (FDR) $<0.05$ (Fig. 1a).

Additionally, to further the investigate potential functions of the differentially expressed IRGs in male GC patients, GO and KEGG analyses were performed in $\mathrm{R}$ software. As shown in Fig. 1b, the results of GO enrichment analysis indicated that these IRGs can be significantly enriched in several important immune responses, including cell chemotaxis, leukocyte chemotaxis, myeloid leukocyte migration, leukocyte migration, granulocyte chemotaxis, neutrophil chemotaxis, granulocyte

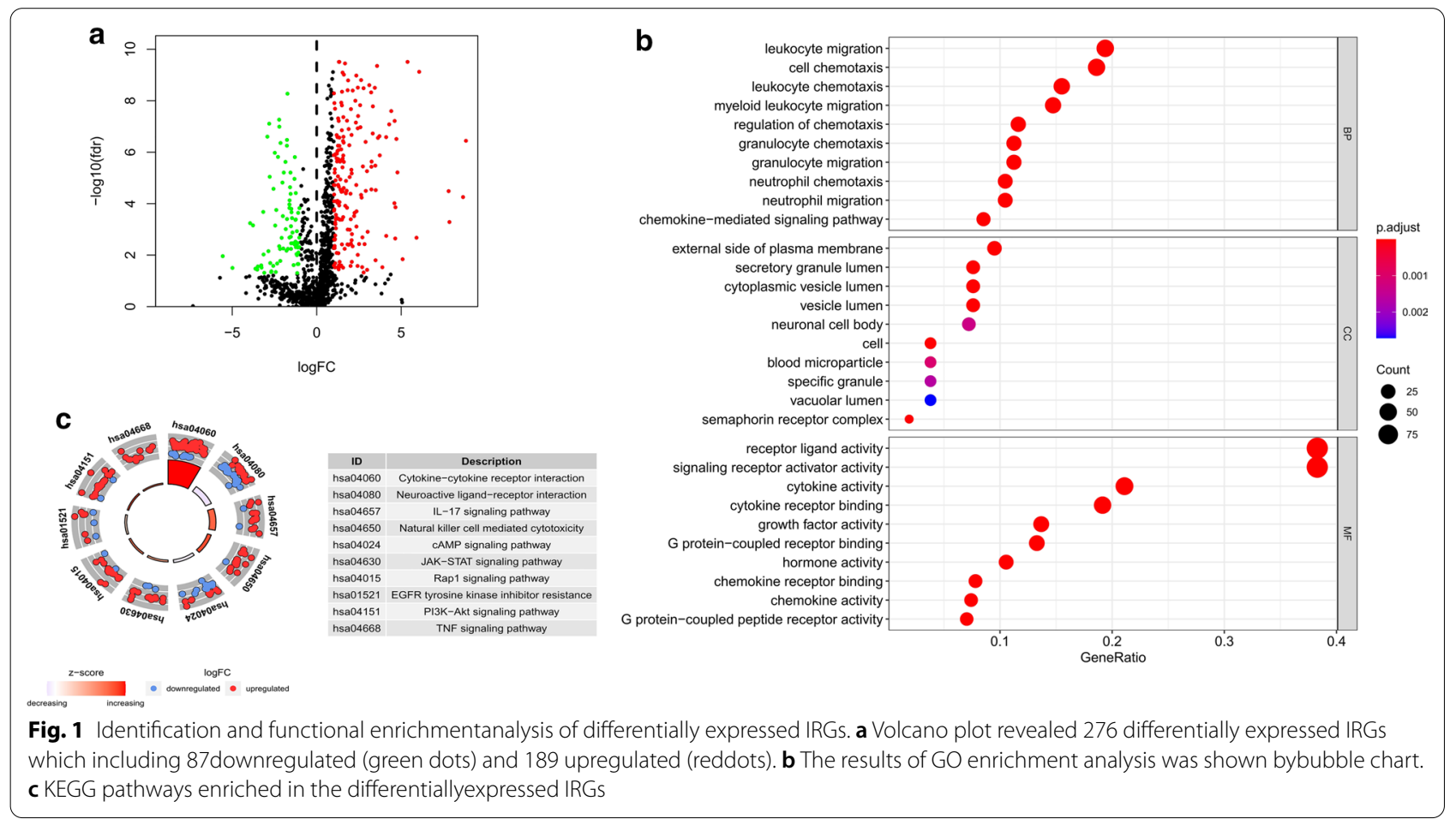


migration and neutrophil migration. KEGG analysis highlighted that the differentially expressed IRGs were mainly enriched in immune responses and tumourrelated signalling pathways (Fig. 1c).

\section{Identification of OS-related IRGs}

A total of 20 differentially expressed IRGs were identified to be significantly associated with the OS of male GC patients based on the results of univariate Cox regression analysis (Fig. 2a). Then, by performing multivariate Cox regression analysis, a prognostic signature consisting of seven IRGs (LCN12, CCL21, RNASE2, CGB5, NRG4, AGTR1 and NPR3) was selected to construct a prediction model (Table 1). All the seven IRGs were associated with high risk with hazard ratios (HRs) $>1$. Among these IRGs, three genes (LCN12, RNASE2, and CGB5) were upregulated and four genes (CCL21, NRG4, AGTR1, and NPR3) were downregulated in male GC tissues compared to the normal tissues based on the TCGA dataset (Fig. 2b).

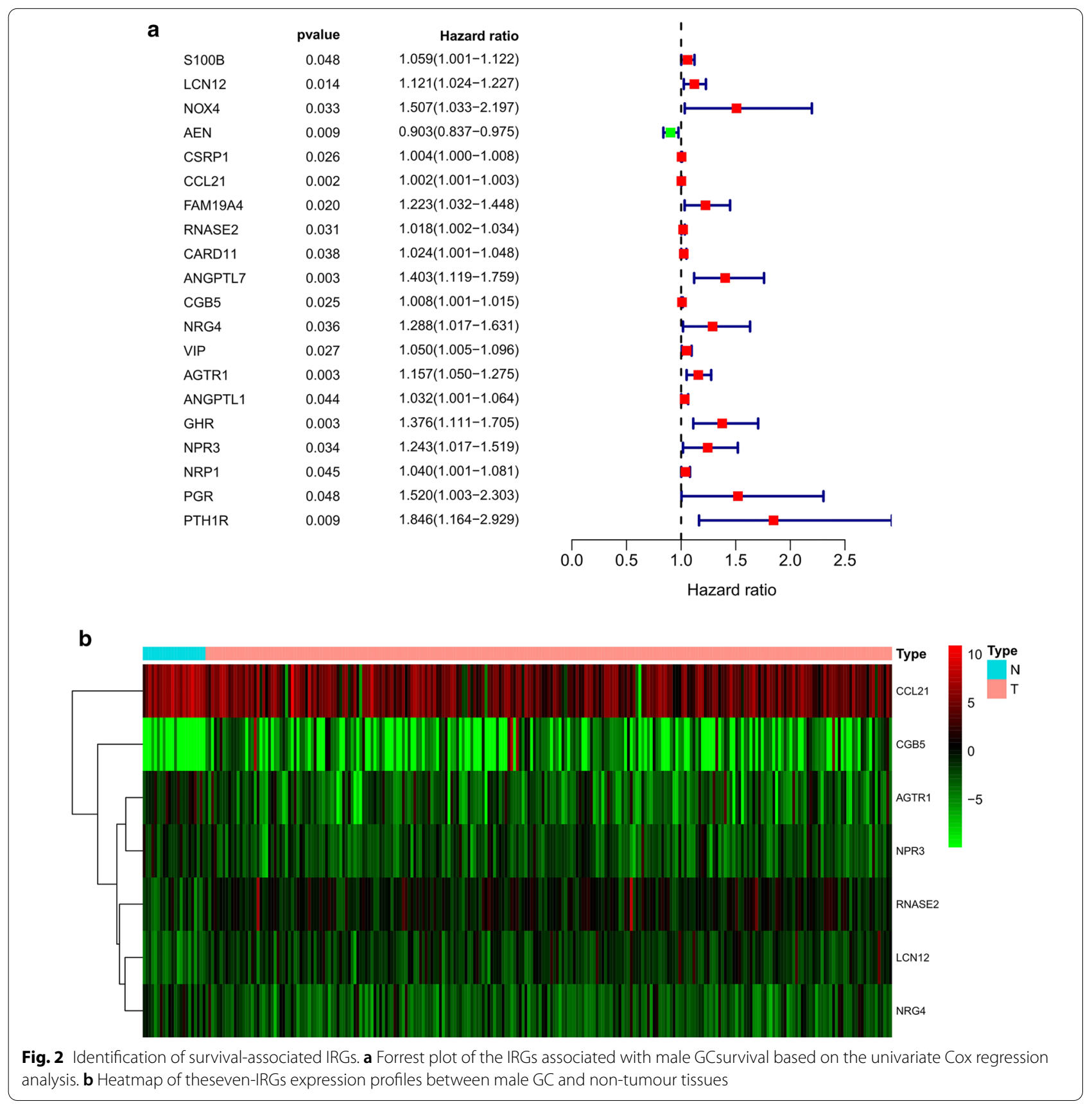


Table 1 The detail information of the seven-IRGs risk signature based on multivariate Cox regression analysis

\begin{tabular}{|c|c|c|c|c|}
\hline Gene & Full name & Coefficient & HR & $P$ value \\
\hline LCN12 & Lipocalin 12 & 0.13933 & 1.14950 & 0.00114 \\
\hline CCL21 & Chemokine (C-C motif) ligand 21 & 0.00181 & 1.00181 & 0.00263 \\
\hline RNASE2 & Ribonuclease a family member 2 & 0.01554 & 1.01566 & 0.05666 \\
\hline CGB5 & Chorionic gonadotropin subunit beta 5 & 0.01036 & 1.01041 & 0.00464 \\
\hline NRG4 & Neuregulin 4 & 0.34521 & 1.41229 & 0.00552 \\
\hline AGTR1 & Angiotensin II receptor type 1 & 0.14512 & 1.15618 & 0.00545 \\
\hline NPR3 & Natriuretic peptide receptor 3 & 0.23201 & 1.26113 & 0.03554 \\
\hline
\end{tabular}

\section{Establishment of the seven-IRGs risk signature}

Subsequently, we constructed a prognostic model on the basis of the seven-IRGs. The risk score of each male GC patient was calculated as follows: risk score $=$ expression level of $\mathrm{LCN} 12 \times 0.13933+$ expression level of CCL21 $\times 0.00181+$ expression level of RNASE $2 \times 0.01554+$ expression level of CGB5 $\times 0.01036+$ expression level of NRG4 $\times 0.34521+$ expression level of AGTR1 $\times 0.14512+$ expression level of NPR3 $\times 0.23201$. The male GC patients were classified into high- and lowrisk groups according to the median risk score (Additional file 1). The distribution of risk scores and the survival status of male GC patients were displayed in Fig. 3a. In addition, the heatmap revealed the differentially expressed levels of the seven-IRGs in the high- and low-risk groups (Fig. 3b).

Next, the prognostic value of the risk score was evaluated. Univariate Cox regression analysis showed that the risk score $(P<0.001, \mathrm{HR}=1.268,95 \%$ CI $1.151-1.397)$ was significantly correlated with the OS of male GC patients (Fig. 4a). Notably, as shown in Fig. 4b, the risk score could be an independent prognostic indicator $(P<0.001, \mathrm{HR}=1.288,95 \%$ CI 1.167-1.422). The KaplanMeier curve demonstrated that male GC patients with high risk scores had a shorter survival time than those with low risk scores (log-rank $P<0.001$, Fig. 4c). The areas under the curve (AUCs) for the risk score at 1-, 3and 5-year in predicting OS were 0.73, 0.633 and 0.745 , respectively (Fig. 4d). Moreover, compared to other clinical parameters, the risk score had the highest performance in the survival prediction of male GC patients $(\mathrm{AUC}=0.712$, Fig. $4 \mathrm{e})$. Taken together, the above results indicated that the risk score performed well at predicting OS in the TCGA dataset.

\section{Construction of the prognostic nomogram}

To better predict the prognosis of male GC patients, we established a nomogram to predict the OS probability at 1-, 3- and 5-years (Fig. 5a). The variables of age, grade, stage and risk score were included in the prediction model. The C-index of the nomogram was 0.695 (95\% CI 0.632-0.759). As shown in Fig. 5b, the calibration plots demonstrated favourable agreement between predicted probabilities from the nomogram and the observed outcomes. Collectively, these results implied that the nomogram had good reliability in predicting survival for male GC patients.

External validation of the seven-IRGs prognostic signature To further examine the performance of the seven IRGsbased model, the gene expression data and survival outcomes from GSE15460 were used for external validation. We calculated the risk score with the same formula for each male GC patient, and then divided them into highand low-risk groups according to the median risk score (Additional file 2). Compared with patients in the lowrisk group, male GC patients with high risk scores suffered significantly more survival risks (Fig. 6a). Similar to the abovementioned findings, male GC patients in the high-risk group were associated with poor survival outcomes (log-rank $P=0.001$, Fig. $6 \mathrm{~b}$ ). As presented in Fig. 6c, the AUCs of 1-, 3- and 5-year in predicting OS were $0.595,0.621$ and 0.657 , respectively. Furthermore, the calibration plots indicated quite good agreement between prediction and observation for the 3- and 5-year OS probabilities of the patients (Fig. 6d).

\section{Associations of the risk signature with tumour-infiltrating immune cells}

To investigate distinct patterns of immune infiltration, we used CIBERSORT algorithm to estimate the composition of 22 infiltrating immune cells types in each male GC sample. As presented by the radar plot in Fig. 7a, the abundance of the 22 infiltrative immune cells was significantly different between high- and low-risk groups. Specifically, the infiltration levels of resting memory CD4 T cells $(P=0.034)$, activated NK cells $(P=0.003)$, regulatory $\mathrm{T}$ cells (Tregs) $(P=0.002)$, monocytes $(P=0.004)$ and resting mast cells $(P=0.008)$ were significantly higher in the high-risk group than in the low-risk group, whereas 

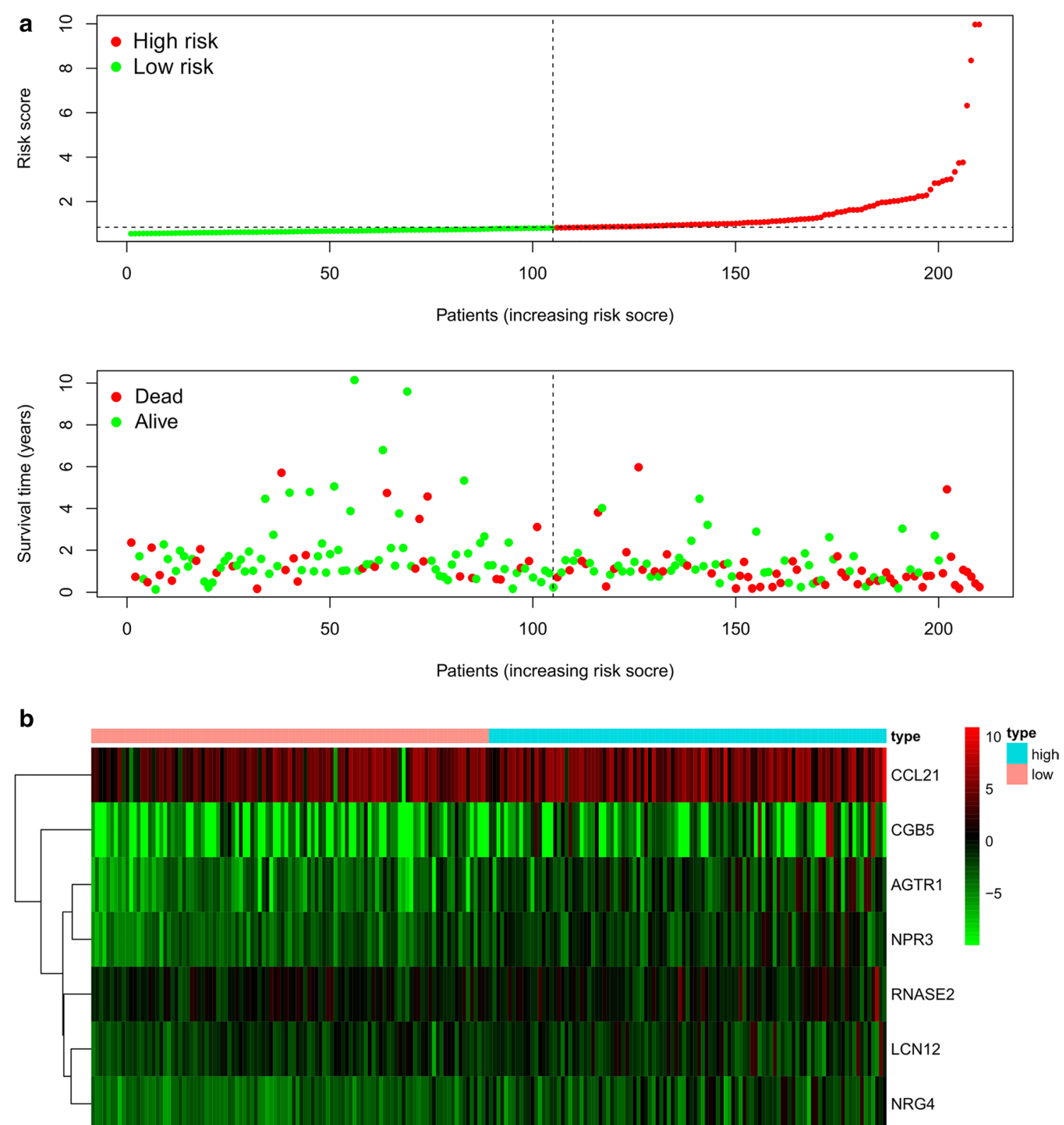

Fig. 3 Characteristics of the seven-IRGs signature inthe TCGA dataset. a The distribution of riskscore andthe survival status of male GC patients. The dotted line is the optimal cut-off value for dividing male GC patients intohigh- and low-risk groups. $\mathbf{b}$ Heatmap of the seven-IRGs expression profilesbetween the high- and low-risk groups

the infiltration levels of activated memory CD4 $\mathrm{T}$ cells $(P<0.001)$, resting NK cells $(P<0.001)$, follicular helper T cells $(P=0.009)$ and M1 macrophages $(P=0.006)$ were the opposite (Fig. 7b).

\section{Discussion}

Although the morbidity and mortality of GC have declined over the past decade [21], we still face many problems and challenges in the screening and treatment of GC. Because of sex differences and tumour heterogeneity, even patients with the same pathologic stage may have considerable differences in survival, indicating that prognosis cannot be accurately determined based on the current staging system alone [22]. Epidemiological data and studies have pointed out that males suffer a higher risk and poorer prognosis than females in many types of cancer [23, 24]. Finding new predictors with good prognostic value for male GC patients is urgently 


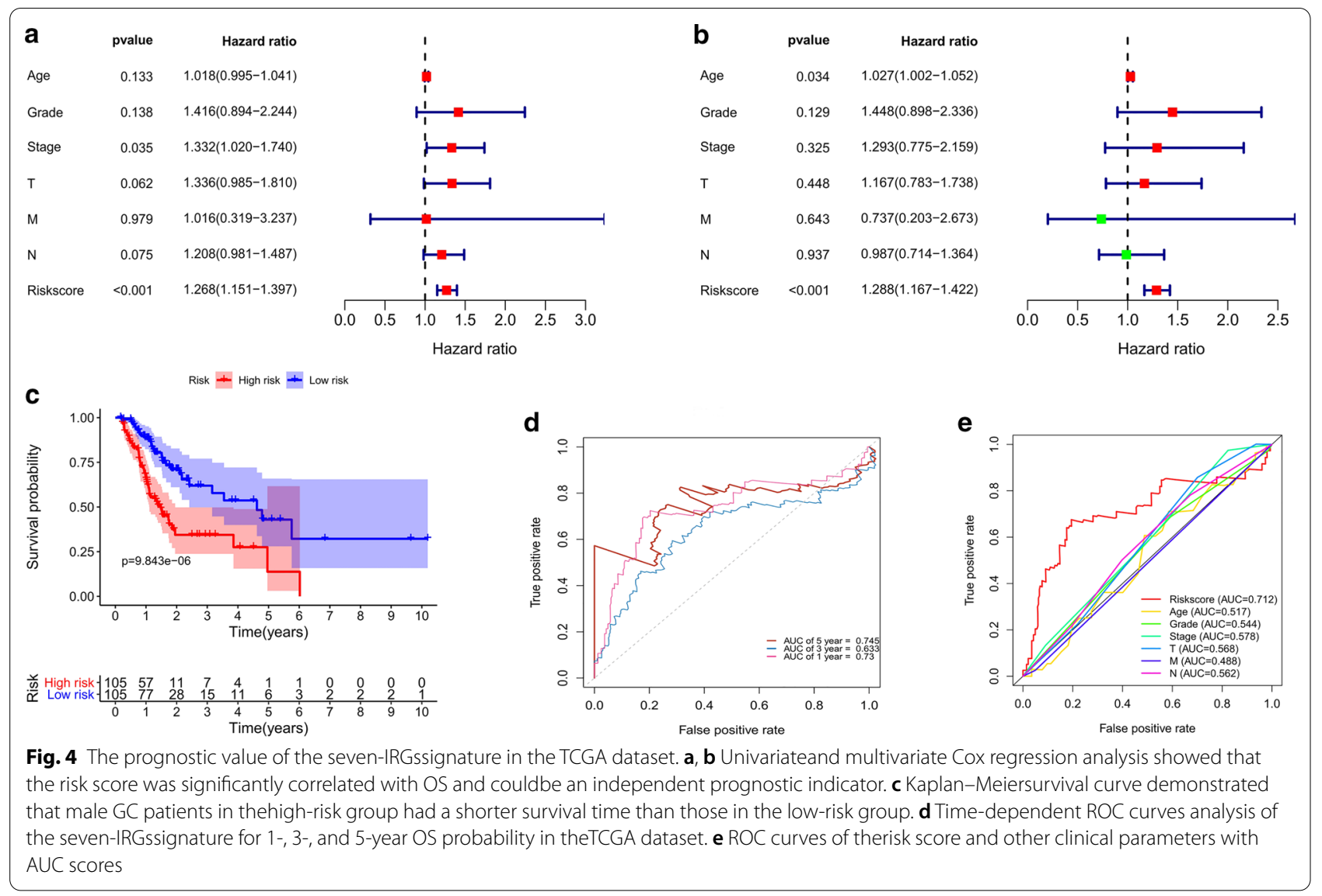

needed, which is also in line with the development of precision medicine.

The role of the immune system in cancer is twofold: it not only suppresses tumour growth but also promotes tumour progression [25]. Because of the complexity of immune response and tumour biology, it is difficult to predict the survival of patients only by a single biomarker. In this study, we first screened 276 differentially expressed IRGs between male GC and non-tumour tissues. Bioinformatics enrichment analysis demonstrated that these IRGs were mainly related to immune responses and several tumour-related signalling pathways. Then, based on the results of univariate and multivariate Cox regression analysis, an immune related risk score model for male GC patients, which is composed of seven differentially expressed IRGs, was constructed using TCGA dataset. Male GC patients with high risk scores had significantly poorer OS than those with low risk scores. The risk score could be an independent prognostic indicator for male GC patients. Moreover, the prognostic value of IRGs signature was further validated in an external dataset.

Among the seven IRGs, four IRGs (CCL21, CGB5, NRG4 and AGTR1) have been reported to be involved in the development and progression of GC. Concretely speaking, Tang et al. reported that CCL21 is overexpressed in Epstein-Barr virus-associated GC and protects $\mathrm{CD} 8^{+} \mathrm{CCR} 7^{+} \mathrm{T}$ lymphocytes from apoptosis via the mitochondria-mediated pathway [26]. In addition, high expression of CCL21 was related to lymph node metastasis and poor prognosis in GC patients [27]. CGB5 was found to be overexpressed in most GC tissues and was considered as an independent predictor of OS and recurrence-free survival for GC patients [28]. As the specific ligand of HER4, the role of NRG4 in GC remains controversial. A previous study demonstrated both HER4 and NRG4 were downregulated in GC tissues compared to matched normal tissues [29]. In contrast, another study indicated that HER4 was overexpression in GC, but not associated with survival [30]. Notably, the NRG4-HER4 axis might also play an important role in the proliferation of malignant lymphoma cells in the gastrointestinal tract [31]. In GC, the utility of AGTR1 inhibitor significantly suppresses GC cell proliferation and stromal fibrosis [32]. It has been proven that AGTR1 could enhance malignant phenotype of several cancer cells to promote tumour progression [33-35]. The roles of RNASE2 and NPR3 in GC have not been reported. A recent study figured out that 


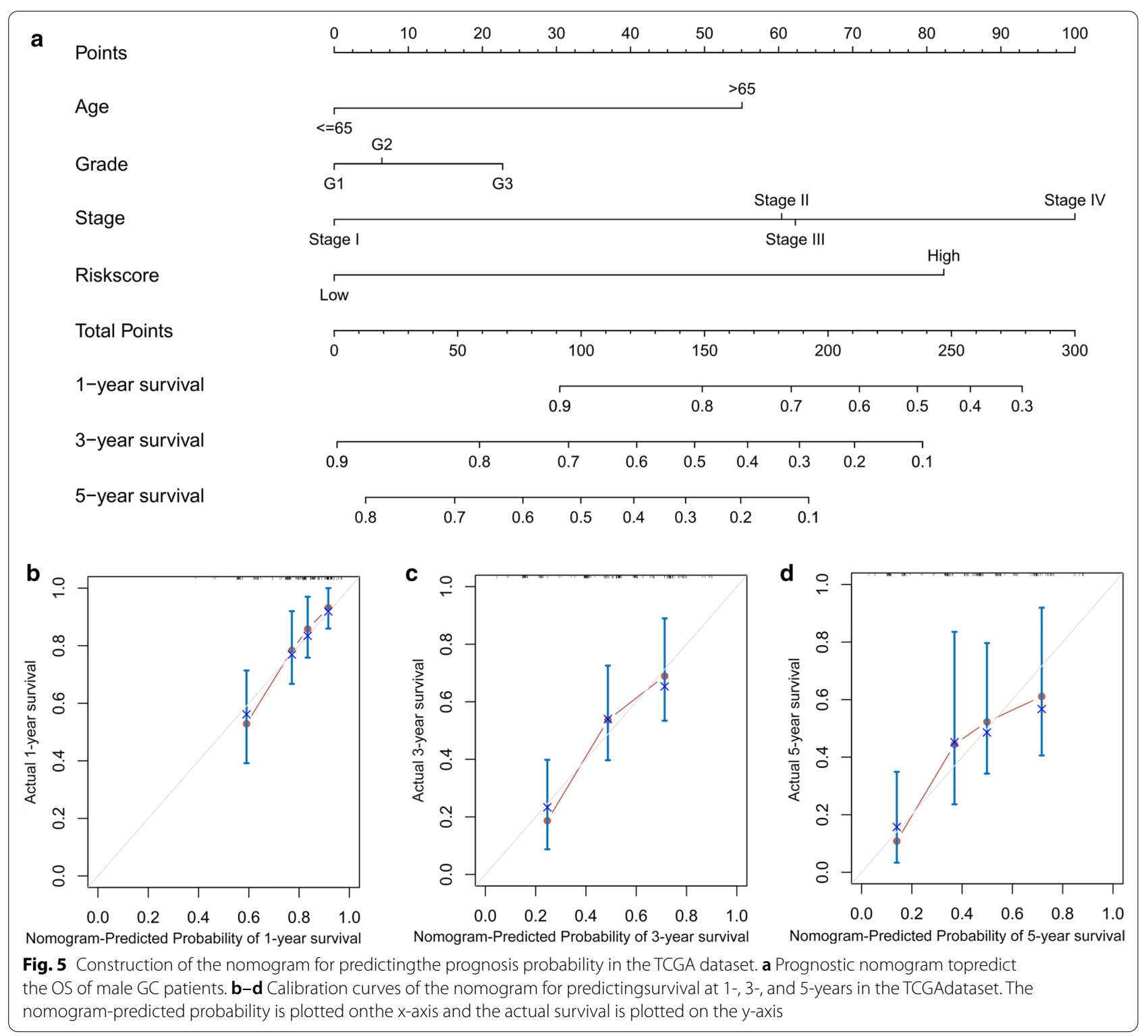

RNASE2 could be a valuable prognostic predictor in clear cell renal cell carcinoma [36]. Moreover, the expression of RNASE2 was significantly upregulated in childhood acute lymphoblastic leukaemia [37]. Li et al. reported that MRCCAT1 promotes metastasis of clear cell renal cell carcinoma via inhibiting NPR3 expression [38]. However, in hepatocellular carcinoma, FENDRR promoted cancer cells apoptosis by targeting miR-362-5p via stimulating NPR3 expression [39]. In addition, NPR3 upregulation could also promote the proliferation of colorectal cancer cells [40]. There are few studies about LCN12, and its role in cancer has not yet been elucidated. LCN12 belongs to the lipocalin family of proteins, which is associated with male reproduction and immune response [41, 42].
In addition, a nomogram combined with our risk signature model and clinicopathological parameters was developed to predict the 1-, 3- and 5-year OS of male GC patients. To our knowledge, the seven-IRGs signature related prognostic model and nomogram have not been reported to date. This nomogram could provide an intuitive visual presentation of individualized survival prediction for both doctors and patients.

Finally, given the critical role of tumour-infiltrating immune cells in tumour progression, the difference of the composition of 22 infiltrating immune cells types in the high- and low-risk groups was analysed. We found that the percentages of resting memory CD4 $\mathrm{T}$ cells, activated NK cells, Tregs, monocytes and resting mast cells 

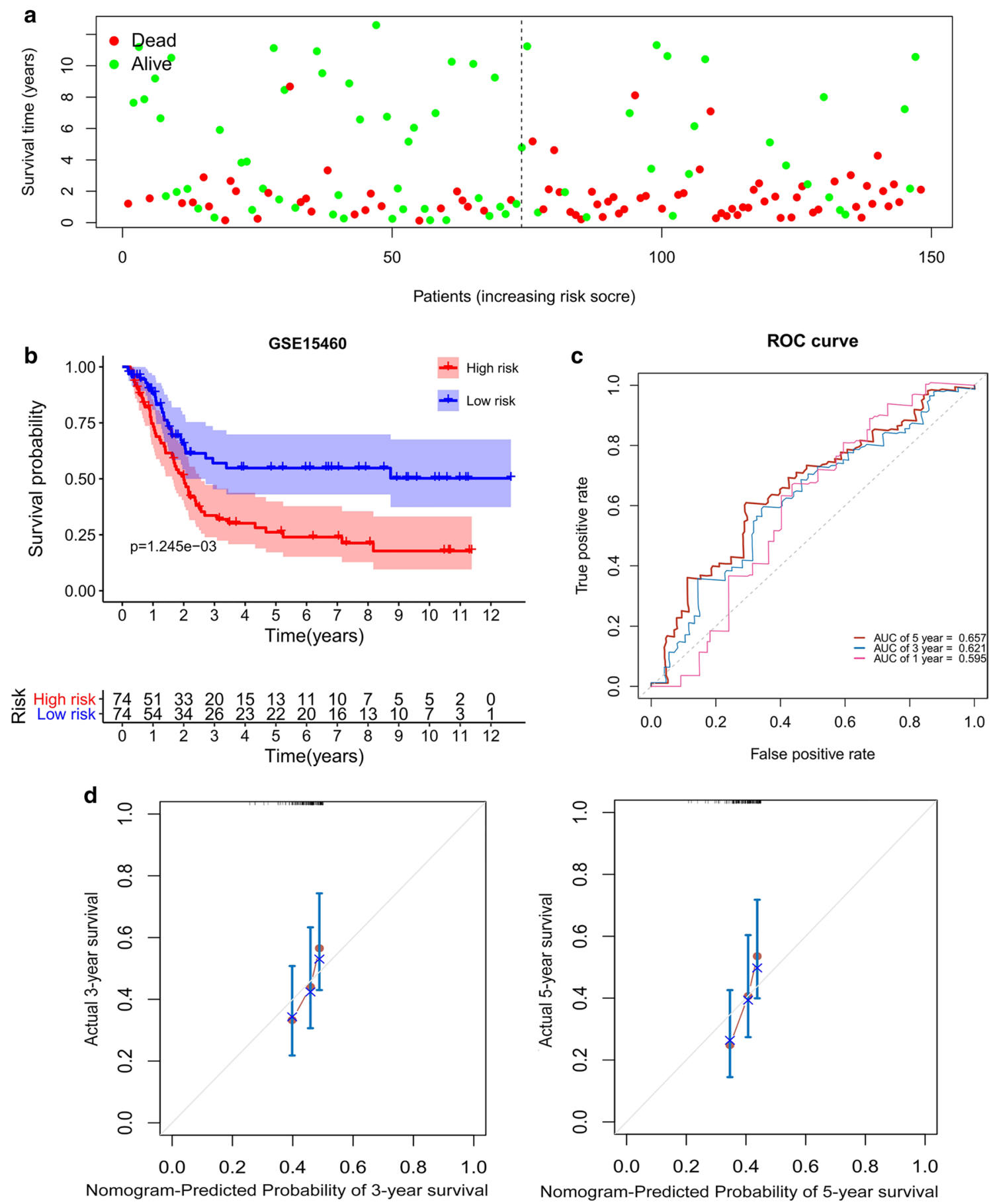

Fig. 6 External validation of the seven-IRGsprognostic risk model. a The survival status and time of male GC patientsdistributed by risk score in GSE15460. The dotted line isthe optimal cut-off value for dividing male GC patients into high- and low-riskgroups. b Kaplan-Meier survival curve of male GC patients in the high- and low-risk groups in GSE15460. c Time-dependent ROC curves analysis of the seven-IRGssignature for 1-, 3-, and 5-year OS probability in GSE15460. d Calibration curves ofthe nomogram for predicting survival at 3-, and 5-years in GSE15460. Thenomogram-predicted probability is plotted on the $x$-axis and the actual survivalis plotted on the $y$-axis 

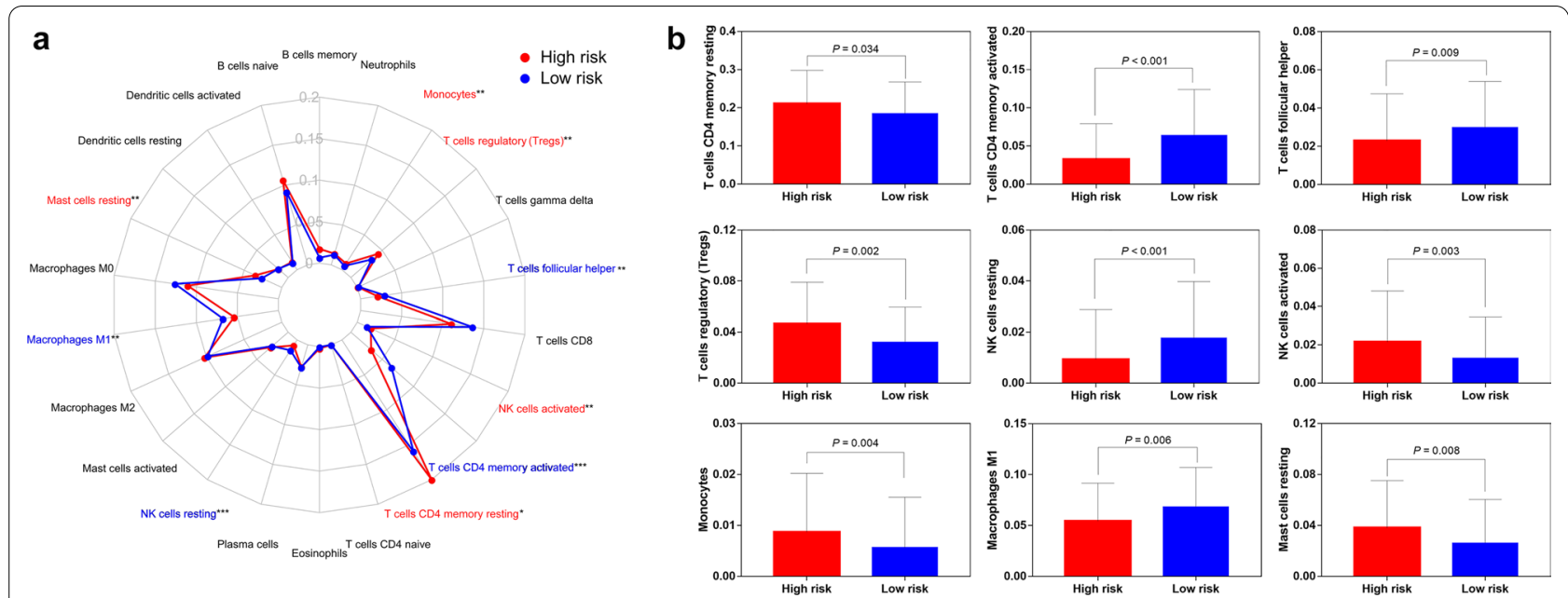

Fig. 7 Associations of the seven-IRGs signature with tumour-infiltrating immune cells in TCGA dataset. a Differentialdistribution of 22 infiltrating immune cell types between high- and low-risk groups. b The bar chart exhibited the significantinfiltrating difference of several immune cells in the high- and low-risk groups. ${ }^{*} P<0.05,{ }^{* *} P<0.01,{ }^{* * *} P<0.001$

were significantly higher in the high-risk group, while activated memory CD4 $\mathrm{T}$ cells, resting NK cells, follicular helper T cells and M1 macrophages were mainly enriched in the low-risk group. This result was basically consistent with previous studies. For instance, a recent study revealed that resting memory $\mathrm{CD} 4 \mathrm{~T}$ cells was one of the most abundant tumour-infiltrating immune cells in GC samples; in addition, the infiltration levels of activated memory CD4 $\mathrm{T}$ cells was positively correlated with a favourable prognosis for GC patients [43]. It was reported that $\mathrm{GC}$ patients with high infiltration of FOXP3(+) Tregs exhibited a lower OS rate and a poor outcome [44]. In the TME, macrophages could display antitumour M1 and protumour M2 phenotypes, and high density of M1 macrophages was associated with better OS in GC patients [45, 46]. Follicular helper $\mathrm{T}$ cells have been discovered in tertiary lymphoid structures of several tumours, suggesting that they might play a vital role in the generation of effective and durable antitumour immune responses [47-49]. Additionally, mast cells and monocytes act as proinflammatory, angiogenic, lymphangiogenic and immunomodulatory mediator in GC progression [50]. Indeed, we also noticed that tumourassociated monocytes/macrophages could impair NK cells function through TGF $\beta 1$ to promote GC immune escape [51]. Hence, the seven-IRGs risk signature might reflect a changing TME for male GC patients in the highrisk group. Collectively, these results could at least partially explain the poorer outcome for the high-risk group.

However, the current study has several limitations that should be taken into consideration. First, since our study object was only male GC patients, the enrolled and validated sample size was relatively small. Second, more large-scale, multicenter and prospective clinical cohorts are needed to verify the predictive value of the sevenIRGs signature. Third, further experimental studies may also be needed to elucidate underlying the molecular mechanisms of the seven-IRGs in GC.

\section{Conclusions}

In summary, we identified a seven-IRGs signature and established a reliable prognostic nomogram model for OS prediction in male GC patients.

\section{Supplementary Information}

The online version contains supplementary material available at https://doi. org/10.1186/s12935-021-01823-0.

Additional file 1. The detail of risk score for each male GC patient in TCGA dataset.

Additional file 2. The detail of risk score for each male GC patient in GSE15460.

\section{Abbreviations}

GC: Gastric cancer; IRGs: Immune-related genes; TME: Tumourmicroenvironment; GEO: Gene Expression Omnibus; TCGA: The Cancer Genome Atlas; ROC: Receiver operatingcharacteristic; GO: Gene ontology; KEGG: Kyoto Encyclopedia of Genes and Genomes; HR: Hazard ratios; AUC: Area under the curve; OS: Overall survival.

\section{Acknowledgements}

Not applicable.

\section{Authors' contributions}

$X X$ and $Y L$ concepted and designed the study; XX, MW, YLu and HW contributed to date acquisition; XX, YW, XW and $B C$ analyzed data; $X X$ and HW wrote the manuscript. All authors read and approved the final manuscript. 


\section{Funding}

This work was supported by grants from the National Natural Science Foundation of China (NO.81874063).

\section{Availability of data and materials \\ Not applicable.}

\section{Ethics approval and consent to participate}

Not applicable.

\section{Consent for publication}

Not applicable.

\section{Competing interests}

The authors declare that they have no competing interests.

\section{Author details}

${ }^{1}$ Department of General Surgery, The First Affiliated Hospital of Anhui Medical University, 218 JiXi Avenue, Hefei 230022, Anhui, China. ${ }^{2}$ Anhui Medical University, Hefei 230022, China.

Received: 1 August 2020 Revised: 6 January 2021 Accepted: 9 February 2021

Published online: 18 February 2021

\section{References}

1. Li H, Wang C, Wei Z, Chen W, Guo Z, He Y, Zhang C. Differences in the prognosis of gastric cancer patients of different sexes and races and the molecular mechanisms involved. Int J Oncol. 2019:55(5):1049-68.

2. Li H, Wei Z, Wang C, Chen W, He Y, Zhang C. Gender differences in gastric cancer survival: 99,922 cases based on the SEER database. J Gastrointest Surg. 2019;24:1747-57.

3. Wilson MA, Buetow KH. Novel mechanisms of cancer emerge when accounting for sex as a biological variable. Cancer Res. 2020;80(1):27-9.

4. Hanahan D, Weinberg RA. Hallmarks of cancer: the next generation. Cell. 2011;144(5):646-74.

5. Li B, Chan HL, Chen P. Immune checkpoint inhibitors: basics and challenges. Curr Med Chem. 2019;26(17):3009-25.

6. Voena C, Chiarle R. Advances in cancer immunology and cancer immunotherapy. Discov Med. 2016;21(114):125-33.

7. Tamborero D, Rubio-Perez C, Muinos F, Sabarinathan R, Piulats JM, Muntasell A, Dienstmann R, Lopez-Bigas N, Gonzalez-Perez A. A pan-cancer landscape of interactions between solid tumors and infiltrating immune cell populations. Clin Cancer Res. 2018;24(15):3717-28.

8. Thorsson V, Gibbs DL, Brown SD, Wolf D, Bortone DS, Ou Yang TH, PortaPardo E, Gao GF, Plaisier CL, Eddy JA, et al. The immune landscape of cancer. Immunity. 2018;48(4):812-30 e814.

9. Palomero L, Galvan-Femenia I, de Cid R, Espin R, Barnes DR, Blommaert E, Gil-Gil M, Falo C, Stradella A, CIMBA, et al. Immune cell associations with cancer risk iscience. 2020;23(7):101296.

10. Qu Y, Cheng B, Shao N, Jia Y, Song Q, Tan B, Wang J. Prognostic value of immune-related genes in the tumor microenvironment of lung adenocarcinoma and lung squamous cell carcinoma. Aging. 2020;12(6):4757-77.

11. Yao Y, Yan Z, Lian S, Wei L, Zhou C, Feng D, Zhang Y, Yang J, Li M, Chen Y. Prognostic value of novel immune-related genomic biomarkers identified in head and neck squamous cell carcinoma. J Immunother Cancer. 2020;8(2):e000444.

12. Wu J, Zhao Y, Zhang J, Wu Q, Wang W. Development and validation of an immune-related gene pairs signature in colorectal cancer. Oncoimmunology. 2019;8(7):1596715

13. Lin P, Guo YN, Shi L, Li XJ, Yang H, He Y, Li Q, Dang YW, Wei KL, Chen G. Development of a prognostic index based on an immunogenomic landscape analysis of papillary thyroid cancer. Aging. 2019;11(2):480-500.

14. Zhang M, Wang X, Chen X, Zhang Q, Hong J. Novel immune-related gene signature for risk stratification and prognosis of survival in lower-grade glioma. Front Genet. 2020;11:363.
15. Shen C, Liu J, Wang J, Zhong X, Dong D, Yang X, Wang Y. Development and validation of a prognostic immune-associated gene signature in clear cell renal cell carcinoma. Int Immunopharmacol. 2020;81:106274.

16. Jiang B, Sun Q, Tong Y, Wang Y, Ma H, Xia X, Zhou Y, Zhang X, Gao F, Shu P. An immune-related gene signature predicts prognosis of gastric cancer. Medicine. 2019;98(27):e16273.

17. Bhattacharya S, Andorf S, Gomes L, Dunn P, Schaefer H, Pontius J, Berger P, Desborough V, Smith T, Campbell J, et al. ImmPort: disseminating data to the public for the future of immunology. Immunol Res. 2014;58(2-3):234-9.

18. Ritchie ME, Phipson B, Wu D, Hu Y, Law CW, Shi W, Smyth GK. limma powers differential expression analyses for RNA-sequencing and microarray studies. Nucleic Acids Res. 2015;43(7):e47.

19. Wang Z, Gao L, Guo X, Feng C, Lian W, Deng K, Xing B. Development and validation of a nomogram with an autophagy-related gene signature for predicting survival in patients with glioblastoma. Aging. 2019;11(24):12246-69.

20. Newman AM, Liu CL, Green MR, Gentles AJ, Feng W, Xu Y, Hoang CD, Diehn M, Alizadeh AA. Robust enumeration of cell subsets from tissue expression profiles. Nat Methods. 2015;12(5):453-7.

21. Bray F, Ferlay J, Soerjomataram I, Siegel RL, Torre LA, Jemal A. Global cancer statistics 2018: GLOBOCAN estimates of incidence and mortality worldwide for 36 cancers in 185 countries. CA Cancer J Clin. 2018;68(6):394-424.

22. Zeng D, Zhou R, Yu Y, Luo Y, Zhang J, Sun H, Bin J, Liao Y, Rao J, Zhang Y, et al. Gene expression profiles for a prognostic immunoscore in gastric cancer. Br J Surg. 2018;105(10):1338-48.

23. Zhu $Y$, Shao $X$, Wang $X$, Liu L, Liang $H$. Sex disparities in cancer. Cancer Lett. 2019;466:35-8.

24. Zheng D, Trynda J, Williams C, Vold JA, Nguyen JH, Harnois DM, Bagaria SP, McLaughlin SA, Li Z. Sexual dimorphism in the incidence of human cancers. BMC Cancer. 2019;19(1):684.

25. Schreiber RD, Old LJ, Smyth MJ. Cancer immunoediting: integrating immunity's roles in cancer suppression and promotion. Science. 2011:331(6024):1565-70.

26. Tang F, Chen JN, Zhang NN, Gong LP, Jiang Y, Feng ZY, Xiao L, Li HG, Du $H$, Wu $B$, et al. Expression of CCL21 by EBV-associated gastric carcinoma cells protects CD8(+)CCR7(+) T lymphocytes from apoptosis via the mitochondria-mediated pathway. Pathology. 2018;50(6):613-21.

27. Hwang TL, Lee LY, Wang CC, Liang Y, Huang SF, Wu CM. CCL7 and CCL21 overexpression in gastric cancer is associated with lymph node metastasis and poor prognosis. World J Gastroenterol. 2012;18(11):1249-56.

28. Yang Y, Shi Y, Hou Y, Lu Y, Yang J. CGB5 expression is independently associated with poor overall survival and recurrence-free survival in patients with advanced gastric cancer. Cancer Med. 2018;7(3):716-25.

29. Nielsen TO, Friis-Hansen L, Poulsen SS, Federspiel B, Sorensen BS. Expression of the EGF family in gastric cancer: downregulation of HER4 and its activating ligand NRG4. PLOS ONE. 2014;9(4):e94606.

30. Yun S, Koh J, Nam SK, Park JO, Lee SM, Lee K, Lee KS, Ahn SH, Park DJ, $\mathrm{Kim} \mathrm{HH}$, et al. Clinical significance of overexpression of NRG1 and its receptors, HER3 and HER4, in gastric cancer patients. Gastric Cancer. 2018;21(2):225-36.

31. Ebi M, Kataoka H, Shimura T, Hirata Y, Mizushima T, Mizoshita T, Tanaka M, Tsukamoto H, Ozeki K, Tanida S, et al. The role of neuregulin4 and HER4 in gastrointestinal malignant lymphoma. Mol Med Rep. 2011;4(6):1151-5.

32. Okazaki M, Fushida S, Harada S, Tsukada T, Kinoshita J, Oyama K, Tajima H, Ninomiya I, Fujimura T, Ohta T. The angiotensin II type 1 receptor blocker candesartan suppresses proliferation and fibrosis in gastric cancer. Cancer Lett. 2014;355(1):46-53.

33. Fujimoto Y, Sasaki T, Tsuchida A, Chayama K. Angiotensin II type 1 receptor expression in human pancreatic cancer and growth inhibition by angiotensin II type 1 receptor antagonist. FEBS Lett. 2001;495(3):197-200.

34. Zhang Q, Yu S, Lam MMT, Poon TCW, Sun L, Jiao Y, Wong AST, Lee LTO. Angiotensin II promotes ovarian cancer spheroid formation and metastasis by upregulation of lipid desaturation and suppression of endoplasmic reticulum stress. J Exp Clin Cancer Res CR. 2019;38(1):116.

35. Ma Y, Xia Z, Ye C, Lu C, Zhou S, Pan J, Liu C, Zhang J, Liu T, Hu T, et al. AGTR1 promotes lymph node metastasis in breast cancer by upregulating CXCR4/SDF-1alpha and inducing cell migration and invasion. Aging. 2019;11(12):3969-92. 
36. Wan B, Liu B, Huang Y, Yu G, Lv C. Prognostic value of immune-related genes in clear cell renal cell carcinoma. Aging. 2019;11(23):11474-89.

37. Niini T, Vettenranta K, Hollmen J, Larramendy ML, Aalto Y, Wikman H, Nagy B, Seppanen JK, Ferrer Salvador A, Mannila H, et al. Expression of myeloidspecific genes in childhood acute lymphoblastic leukemia-a cDNA array study. Leukemia. 2002;16(11):2213-21.

38. Li JK, Chen C, Liu JY, Shi JZ, Liu SP, Liu B, Wu DS, Fang ZY, Bao Y, Jiang MM, et al. Long noncoding RNA MRCCAT1 promotes metastasis of clear cell renal cell carcinoma via inhibiting NPR3 and activating p38-MAPK signaling. Mol Cancer. 2017;16(1):111.

39. Qian G, Jin X, Zhang L. LncRNA FENDRR upregulation promotes hepatic carcinoma cells apoptosis by targeting miR-362-5p via NPR3 and p38MAPK pathway. Cancer Biother Radiopharm. 2020;35:629-39.

40. Gu L, Lu L, Zhou D, Liu Z. Long noncoding RNA BCYRN1 promotes the proliferation of colorectal cancer cells via up-regulating NPR3 expression. Cell Physiol Biochem. 2018;48(6):2337-49.

41. Suzuki K, Lareyre JJ, Sanchez D, Gutierrez G, Araki Y, Matusik RJ, OrgebinCrist MC. Molecular evolution of epididymal lipocalin genes localized on mouse chromosome 2. Gene. 2004;339:49-59.

42. Schiefner A, Skerra A. The menagerie of human lipocalins: a natural protein scaffold for molecular recognition of physiological compounds. Acc Chem Res. 2015;48(4):976-85.

43. Li L, Ouyang Y, Wang W, Hou D, Zhu Y. The landscape and prognostic value of tumor-infiltrating immune cells in gastric cancer. PeerJ. 2019;7:e7993.

44. Hou J, Yu Z, Xiang R, Li C, Wang L, Chen S, Li Q, Chen M, Wang L. Correlation between infiltration of FOXP3 + regulatory $T$ cells and expression of $\mathrm{B} 7-\mathrm{H} 1$ in the tumor tissues of gastric cancer. Exp Mol Pathol. 2014;96(3):284-91.

45. Yin S, Huang J, Li Z, Zhang J, Luo J, Lu C, Xu H, Xu H. The prognostic and clinicopathological significance of tumor-associated macrophages in patients with gastric cancer: a meta-analysis. PloS one. 2017;12(1):e0170042.

46. Gambardella V, Castillo J, Tarazona N, Gimeno-Valiente F, MartinezCiarpaglini C, Cabeza-Segura M, Rosello S, Roda D, Huerta M, Cervantes
A, et al. The role of tumor-associated macrophages in gastric cancer development and their potential as a therapeutic target. Cancer Treat Rev. 2020;86:102015.

47. Gu-Trantien C, Loi S, Garaud S, Equeter C, Libin M, de Wind A, Ravoet M, Le Buanec H, Sibille C, Manfouo-Foutsop G, et al. CD4(+) follicular helper T cell infiltration predicts breast cancer survival. J Clin Investig. 2013;123(7):2873-92.

48. Coppola D, Nebozhyn M, Khalil F, Dai H, Yeatman T, Loboda A, Mule JJ. Unique ectopic lymph node-like structures present in human primary colorectal carcinoma are identified by immune gene array profiling. Am J Pathol. 2011;179(1):37-45.

49. de Chaisemartin L, Goc J, Damotte D, Validire P, Magdeleinat P, Alifano M, Cremer I, Fridman WH, Sautes-Fridman C, Dieu-Nosjean MC. Characterization of chemokines and adhesion molecules associated with T cell presence in tertiary lymphoid structures in human lung cancer. Cancer Res. 2011;71(20):6391-9.

50. Sammarco G, Varricchi G, Ferraro V, Ammendola M, De Fazio M, Altomare DF, Luposella M, Maltese L, Curro G, Marone G, et al. Mast cells, angiogenesis and lymphangiogenesis in human gastric cancer. Int J Mol Sci. 2019;20(9):2106.

51. Peng LS, Zhang JY, Teng YS, Zhao YL, Wang TT, Mao FY, Lv YP, Cheng P, Li $\mathrm{WH}$, Chen N, et al. Tumor-associated monocytes/macrophages impair NK-cell function via TGFbeta1 in human gastric cancer. Cancer Immunol Res. 2017;5(3):248-56.

\section{Publisher's note}

Springer Nature remains neutral with regard to jurisdictional claims in published maps and institutional affiliations.
Ready to submit your research? Choose BMC and benefit from:

- fast, convenient online submission

- thorough peer review by experienced researchers in your field

- rapid publication on acceptance

- support for research data, including large and complex data types

- gold Open Access which fosters wider collaboration and increased citations

- maximum visibility for your research: over 100M website views per year

At BMC, research is always in progress.

Learn more biomedcentral.com/submissions 